PROCEEDINGS OF THE

AMERICAN MATHEMATICAL SOCIETY

Volume 137, Number 1, January 2009, Pages 371-380

S 0002-9939(08)09505-1

Article electronically published on July 30, 2008

\title{
COMBINATORIAL DESCRIPTION OF THE HOMOTOPY GROUPS OF WEDGE OF SPHERES
}

\author{
HAO ZHAO AND XIANGJUN WANG
}

(Communicated by Paul Goerss)

\begin{abstract}
In this paper, we give a combinatorial description of the homotopy groups of a wedge of spheres. This result generalizes that of $\mathrm{J}$. Wu on the homotopy groups of a wedge of 2-spheres. In particular, the higher homotopy groups of spheres are given as the centers of certain combinatorially described groups with special generators and relations.
\end{abstract}

\section{INTRODUCTION}

Recall that a simplicial set $K$ is a sequence of sets $K=\left\{K_{0}, K_{1}, \cdots, K_{n}, \cdots\right\}$ together with face operators $d_{i}: K_{n} \rightarrow K_{n-1}$ and degeneracy operators $s_{i}: K_{n} \rightarrow$ $K_{n+1}$ for each $0 \leq i \leq n$. These operators satisfy the well-known simplicial identities [1, 2].

Given a reduced simplicial set $X$ which satisfies $X_{0}=\{*\}$, D. M. Kan 3 gave a loop construction on $X$ as $G X$ which satisfies the extension condition. Its geometric realization $|G X|$ is homotopy equivalent to $\Omega|X|$. For a reduced simplicial set $X$, we can define its homotopy groups as $\pi_{*}(X)=\pi_{*-1}(G X)$. For instance, the $n$-sphere $S^{n}$ is a reduced simplicial set having only two nondegenerate simplices, a vertex * and $n$-simplex $\sigma_{n}$. We define its homotopy group as $\pi_{*}\left(S^{n}\right)=\pi_{*-1}\left(G S^{n}\right)$.

In [4, J. Wu gave a combinatorial description of the homotopy groups of a wedge of 2-spheres. It was the first time that all the homotopy groups of a 2 -sphere were described. In this paper, we will generalize J. Wu's result by combinatorially describing the homotopy groups of a wedge of spheres in various dimensions. In particular, the higher homotopy groups of spheres are the centers of certain combinatorially described groups with special generators and relations.

Before stating our main result, we list some group-theoretical descriptions. We let $G$ be a group and $[x, y]=x^{-1} y^{-1} x y$ for $x, y \in G$.

Definition 1.1. A commutator bracket arrangement of weight $n$ in a group $G$ is a map $\beta^{n}: G^{n} \rightarrow G$ defined inductively. First we define $\beta^{1}=i d_{G}$ and $\beta^{2}\left(a_{1}, a_{2}\right)=$ $\left[a_{1}, a_{2}\right]$ for any $a_{1}, a_{2} \in G$. Suppose that the commutator bracket arrangements of

Received by the editors December 7, 2007.

2000 Mathematics Subject Classification. Primary 55U10; Secondary 55Q40.

Key words and phrases. Simplicial set, simplicial group, homotopy group of sphere.

This project is supported by NSFC, grant No. 10771105 .

(C)2008 American Mathematical Society 
weight $k$ have been defined for $1 \leq k<n$ with $n \geq 3$. A map $\beta^{n}: G^{n} \rightarrow G$ is called a commutator bracket arrangement of weight $n$ if $\beta^{n}$ is the following composite:

$$
G^{n}=G^{k} \times G^{n-k} \stackrel{\beta^{k} \times \beta^{n-k}}{\longrightarrow} G \times G \stackrel{\beta^{2}}{\longrightarrow} G
$$

for some commutator bracket arrangements $\beta^{k}$ and $\beta^{n-k}$ of weight $k$ and $n-k$, respectively, with $1 \leq k<n$.

Remark. $\beta^{n}$ is a notation for some commutator but is not a function of $n$. Given any $a_{1}, a_{2}, \cdots, a_{n} \in G, \beta^{n}\left(a_{1}, a_{2}, \cdots, a_{n}\right) \in G$ has the form of some commutator such as $\left[\left[a_{1}, \cdots, a_{k}\right],\left[a_{k+1}, \cdots, a_{n}\right]\right]$ with $1 \leq k<n$. For example, when $\beta^{3}$ and $\beta^{4}$ run over all of the commutator bracket arrangements of weights 3 and 4 respectively, $\beta^{3}\left(a_{1}, a_{2}, a_{3}\right)$ has the form $\left[a_{1},\left[a_{2}, a_{3}\right]\right]$ or $\left[\left[a_{1}, a_{2}\right], a_{3}\right]$, and $\beta^{4}\left(a_{1}, a_{2}, a_{3}, a_{4}\right)$ has the form $\left[a_{1},\left[a_{2},\left[a_{3}, a_{4}\right]\right]\right],\left[a_{1},\left[\left[a_{2}, a_{3}\right], a_{4}\right]\right],\left[\left[a_{1}, a_{2}\right],\left[a_{3}, a_{4}\right]\right],\left[\left[a_{1},\left[a_{2}, a_{3}\right]\right], a_{4}\right]$ or $\left[\left[\left[a_{1}, a_{2}\right], a_{3}\right], a_{4}\right]$.

Let $\bigvee_{\alpha \in J} S^{n_{\alpha+1}}$ be the wedge of spheres with indent set $J$ and $n-1>n_{\alpha} \geq 1$ for all $\alpha \in J$. Then our main theorem can be stated as follows.

Theorem 1.2. For $n-1>\max \left\{n_{\alpha} \mid \alpha \in J\right\} \geq 1$, the homotopy group $\pi_{n+1}\left(\bigvee_{\alpha \in J} S^{n_{\alpha}+1}\right)$ is isomorphic to the center of the group

$$
F\left\{\xi_{n-n_{\alpha}}^{n_{\alpha}}\left(i_{n_{\alpha}}, \cdots, i_{1}\right)^{(\alpha)} \mid n-1 \geq i_{n_{\alpha}}>\cdots>i_{1} \geq 0, \alpha \in J\right\}
$$

modulo the normal subgroup generated by

$$
\beta^{t}\left(\xi_{n-n_{\alpha_{1}}}^{n_{\alpha_{1}}}\left(i_{n_{\alpha_{1}}}^{(1)}, \cdots, i_{1}^{(1)}\right)^{\left(\alpha_{1}\right) \varepsilon_{1}}, \cdots, \xi_{n-n_{\alpha_{t}}}^{n_{\alpha_{t}}}\left(i_{n_{\alpha_{t}}}^{(t)}, \cdots, i_{1}^{(t)}\right)^{\left(\alpha_{t}\right) \varepsilon_{t}}\right),
$$

where $F\{\}$ is the free group functor, the definition of $\xi_{n-n_{\alpha}}^{n_{\alpha}}\left(i_{n_{\alpha}}, \cdots, i_{1}\right)^{(\alpha)}$ will be given in Section 3 and

(1) $\varepsilon_{j}= \pm 1$ for $1 \leq j \leq t$;

(2) $n-1 \geq i_{n_{\alpha_{s}}}^{(s)}>\cdots>i_{1}^{(s)} \geq-1$ for $1 \leq s \leq t$;

(3) any of $\{-1,0, \cdots, n-1\}$ appears at least once in $\left\{i_{n_{\alpha_{1}}}^{(1)}, \cdots, i_{1}^{(1)}, \cdots, i_{n_{\alpha_{t}}}^{(t)}\right.$, $\left.\cdots, i_{t}^{(1)}\right\}$;

(4) for $t \geq\left[n / \max \left\{n_{\alpha} \mid \alpha \in J\right\}\right]$, $\beta^{t}$ runs over all of the commutator bracket arrangements of weight $t$, where $\left[n / \max \left\{n_{\alpha} \mid \alpha \in J\right\}\right]$ means the nearest integer which is less than $n / \max \left\{n_{\alpha} \mid \alpha \in J\right\}$.

The article is organized as follows. In Section 2, we will give some preliminaries on the simplicial groups and the combinatorial description of the intersections of certain subgroups in free groups. In Section 3, a transformation on the generators of Milnor's construction $\left(F S^{n_{\alpha}}\right)_{n}$ will be given, and the proof of our main theorem will follow in Section 4.

The authors would like to thank the referee for important comments and helpful suggestions.

\section{Preliminaries on simplicial groups and free groups}

A simplicial group $G$ is a simplicial set with every $G_{n}$ a group and both the face operator and the degeneracy operator are group homomorphisms. A simplicial group $G$ is called free if each $G_{n}$ is a free group and the given bases are stable under all degeneracy operators; that is, if $x \in G_{n}$ is a basis element, then $s_{i} x \in G_{n+1}$ is also a basis element for each $0 \leq i \leq n$ (see [3, 5]). The geometric realizations 
of simplicial groups are topological groups in the category of compactly generated weak Hausdorff spaces.

A simplicial group satisfies the extension condition such that its homotopy groups can be defined. Given a simplicial group $G$, its Moore chain complex $\left(\mathscr{N} G, d_{0}\right)$ is defined by $\mathscr{N} G_{n}=\bigcap_{j=1}^{n} \operatorname{Ker}\left(d_{j}: G_{n} \rightarrow G_{n-1}\right)$ together with the differential $d_{0}: \mathscr{N} G_{n} \rightarrow \mathscr{N} G_{n-1}$. Considering the homotopy groups of simplicial groups, the classical Moore theorem states that $\pi_{n}(G) \cong H_{n}\left(\mathscr{N} G, d_{0}\right)$ (see [3, Theorem 3.7]). We define Moore cycles $\mathscr{Z} G_{n}=\bigcap_{j=0}^{n} \operatorname{Ker}\left(d_{j}: G_{n} \rightarrow G_{n-1}\right)$ and Moore boundaries $\mathscr{B} G_{n}=d_{0}\left(\mathscr{N} G_{n+1}\right)$. Then we have $\pi_{n}(G)=\mathscr{Z} G_{n} / \mathscr{B} G_{n}$. Thus in order to determine the homotopy groups of a topological space $X$, we need to complete the following two steps:

(1) Find a suitable simplicial group model for $X$.

(2) Determine the Moore cycles and boundaries of the simplicial group.

Definition 2.1 (4]). A simplicial group $G$ is said to be $r$-centerless if the center $C\left(G_{n}\right)$ is trivial for all $n \geq r$.

In what follows, we give an important theorem which will be needed in the proof of our main theorem.

Theorem $2.2(4)$. Let $G$ be a reduced $r$-centerless simplicial group. Then we have $\pi_{n}(G)=C\left(G_{n} / \mathscr{B} G_{n}\right)$ for $n \geq r+1$.

Now let us consider the intersection of the kernels of projection homomorphisms between free groups. Let $S$ be a set and $T_{j}$ be a subset of $S$ for $1 \leq j \leq k$. Let $\pi_{j}: F\{S\} \rightarrow F\left\{T_{j}\right\}$ be a projection homomorphism defined by

$$
\pi_{j}(x)= \begin{cases}x & \text { if } \quad x \in T_{j} \\ 1 & \text { if } \quad x \in S-T_{j}\end{cases}
$$

where $1 \leq j \leq k$. We construct a subset $A\left(T_{1}, \cdots, T_{k}\right)$ inductively as follows.

First, we define

$A_{T_{1}}=A\left(T_{1}\right)=\left\{\left[\cdots\left[x, y_{1}^{\varepsilon_{1}}\right] \cdots y_{t}^{\varepsilon_{t}}\right] \mid x \in S-T_{1}, y_{1}, \cdots y_{t} \in T_{1}, \varepsilon_{i}= \pm 1\right.$ for $\left.1 \leq i \leq t\right\}$,

where $y_{1}^{\varepsilon_{1}} \cdots y_{t}^{\varepsilon_{t}} \in F\left\{T_{1}\right\}$ runs over all of the reduced words in $F\left\{T_{1}\right\}$ and for $t=0$, $\left[\cdots\left[x, y_{1}^{\varepsilon_{1}}\right] \cdots y_{t}^{\varepsilon_{t}}\right]=x$. Let

$$
T_{2}^{(2)}=\left\{\omega \in A\left(T_{1}\right) \mid \omega=\left[\cdots\left[x, y_{1}^{\varepsilon_{1}}\right] \cdots y_{t}^{\varepsilon_{t}}\right] \text { with } x, y_{j} \in T_{2} \text { for } 1 \leq i \leq t\right\}
$$

and define $A\left(T_{1}, T_{2}\right)=A\left(T_{1}\right)_{T_{2}^{(2)}}$.

Suppose that $A\left(T_{1}, \cdots, T_{j-1}\right)$ has been defined for $3 \leq j \leq k$ such that all of the elements in $A\left(T_{1}, \cdots, T_{j-1}\right)$ are written as certain commutators in $F\{S\}$ in terms of elements in $S$. Let $T_{j}^{(j)}$ be the subset of $A\left(T_{1}, \cdots, T_{j-1}\right)$ defined by

$T_{j}^{(j)}=\left\{\omega \in A\left(T_{1}, \cdots, T_{j-1}\right) \mid \omega=\left[\cdots,\left[x_{1}^{\varepsilon_{1}}, \cdots\right], \cdots, x_{l}^{\varepsilon_{l}}\right]\right.$ with $x_{i} \in T_{j}$ for $\left.1 \leq i \leq l\right\}$, where $\left[\cdots,\left[x_{1}^{\varepsilon_{1}}, \cdots\right], \cdots, x_{l}^{\varepsilon_{l}}\right]$ is the element in $A\left(T_{1}, \cdots, T_{j-1}\right)$ which is written as a commutator. Then let $A\left(T_{1}, \cdots, T_{j}\right)$ be defined by

$$
A\left(T_{1}, \cdots, T_{j}\right)=A\left(T_{1}, \cdots, T_{j-1}\right)_{T_{j}^{(j)}} .
$$

So after finite steps, we can get $A\left(T_{1}, \cdots, T_{k}\right)$. 
Theorem 2.3 (4]). Let $S$ be a set and $T_{j}$ be a subset of $S$ for $1 \leq j \leq k$. Let $\pi_{j}: F\{S\} \rightarrow F\left\{T_{j}\right\}$ be a projection homomorphism for $1 \leq j \leq k$. Then we have

$$
\bigcap_{j=1}^{k} \operatorname{Ker}\left(\pi_{j}\right)=F\left\{A\left(T_{1}, \cdots, T_{k}\right)\right\}
$$

\section{Transformation on Milnor's construction $\left(F S^{n_{\alpha}}\right)_{n}$}

For a pointed simplicial set $K$, the Milnor construction of $K$ is $F K$, which is a free simplicial group model for $\Omega \Sigma|K|$ (see [6]), where $|K|$ is the geometric realization of $K$. For an $n_{\alpha}$-sphere $S^{n_{\alpha}}$ with $n_{\alpha} \geq 1$, its Milnor construction is $F S^{n_{\alpha}}$. By a Tietze transformation (see [7]), for $n>n_{\alpha}$, we will inductively define a family of generators for $\left(F S^{n_{\alpha}}\right)_{n}$ as

$$
\left\{\xi_{n-n_{\alpha}}^{n_{\alpha}}\left(i_{n_{\alpha}}, \cdots, i_{1}\right) \mid n-1 \geq i_{n_{\alpha}}>\cdots>i_{1} \geq 0\right\} .
$$

First, recall that

$$
\left(S^{n_{\alpha}}\right)_{n}= \begin{cases}\{*\} & \text { for } 0 \leq n \leq n_{\alpha}-1 \\ \left\{*, \sigma_{n_{\alpha}}\right\} & \text { for } n=n_{\alpha} \\ \left\{*, s_{j_{n-n_{\alpha}}} \cdots s_{j_{1}} \sigma_{n_{\alpha}} \mid n-1 \geq j_{n-n_{\alpha}}>\cdots>j_{1} \geq 0\right\} & \text { for } n>n_{\alpha} .\end{cases}
$$

For $n>n_{\alpha}$, we let $\left\{i_{n_{\alpha}}, \cdots, i_{1}\right\}=\{0,1, \cdots, n-1\}-\left\{j_{n-n_{\alpha}}, \cdots, j_{1}\right\}$ and let

$$
\xi_{n-n_{\alpha}}^{0}\left(i_{n_{\alpha}}, \cdots, i_{1}\right)=s_{j_{n-n_{\alpha}}} \cdots s_{j_{1}} \sigma_{n_{\alpha}} .
$$

Then we have

$$
\left(F S^{n_{\alpha}}\right)_{n}=F\left\{\xi_{n-n_{\alpha}}^{0}\left(i_{n_{\alpha}}, \cdots, i_{1}\right) \mid n-1 \geq i_{n_{\alpha}}>\cdots>i_{1} \geq 0\right\},
$$

where $n>n_{\alpha}$ and $F\{\}$ is the free group functor.

Suppose that for $0<j \leq n_{\alpha}, \xi_{n-n_{\alpha}}^{j-1}\left(i_{n_{\alpha}}, \cdots, i_{1}\right)$ has been defined and $\left(F S^{n_{\alpha}}\right)_{n}=$ $F\left\{\xi_{n-n_{\alpha}}^{j-1}\left(i_{n_{\alpha}}, \cdots, i_{1}\right) \mid n=i_{n_{\alpha}+1}>n-1 \geq i_{n_{\alpha}}>\cdots>i_{1}>i_{0}=-1\right\}$, where we let $i_{n_{\alpha}+1}=n$ and $i_{0}=-1$. We define

$$
\begin{aligned}
& \xi_{n-n_{\alpha}}^{j}\left(i_{n_{\alpha}}, \cdots, i_{j}, \cdots, i_{1}\right)= \\
& \left\{\begin{array}{l}
\xi_{n-n_{\alpha}}^{j-1}\left(i_{n_{\alpha}}, \cdots, i_{j}, \cdots, i_{1}\right) \\
\xi_{n-n_{\alpha}}^{j-1}\left(i_{n_{\alpha}}, \cdots, i_{j}, \cdots, i_{1}\right) \xi_{n-n_{\alpha}}^{j-1}\left(i_{n_{\alpha}}, \cdots, i_{j}+1, \cdots, i_{1}\right)^{-1}
\end{array} \text { if } \quad i_{j+1}>i_{j}+1 .\right.
\end{aligned}
$$

By an easy verification, we get that

$$
\xi_{n-n_{\alpha}}^{j-1}\left(i_{n_{\alpha}}, \cdots, i_{j}, \cdots, i_{1}\right)=\prod_{k=0}^{i_{j+1}-i_{j}-1} \xi_{n-n_{\alpha}}^{j}\left(i_{n_{\alpha}}, \cdots, i_{j}+k, \cdots, i_{1}\right) .
$$

It follows that

$$
\left(F S^{n_{\alpha}}\right)_{n}=F\left\{\xi_{n-n_{\alpha}}^{j}\left(i_{n_{\alpha}}, \cdots, i_{1}\right) \mid n-1 \geq i_{n_{\alpha}}>\cdots>i_{1} \geq 0\right\} .
$$

So after finite steps, we get

Proposition 3.1. For $n>n_{\alpha}$, we have

$$
\left(F S^{n_{\alpha}}\right)_{n}=F\left\{\xi_{n-n_{\alpha}}^{n_{\alpha}}\left(i_{n_{\alpha}}, \cdots, i_{1}\right) \mid n=i_{n_{\alpha}+1}>n-1 \geq i_{n_{\alpha}}>\cdots>i_{1}>i_{0}=-1\right\} .
$$

Now let us consider the face operators on the above generators. For any $0 \leq s \leq$ $n=i_{n_{\alpha}+1}$, there must be $a \leq t \leq n_{\alpha}$ such that $i_{t+1} \geq s \geq i_{t}+1$. We have 
Proposition 3.2. For $1 \leq s \leq n=i_{n_{\alpha}+1}$, we have

$$
\begin{aligned}
& d_{s} \xi_{n-n_{\alpha}}^{n_{\alpha}}\left(i_{n_{\alpha}}, \cdots, i_{t+1}, i_{t}, \cdots, i_{1}\right) \\
& = \begin{cases}\xi_{n-n_{\alpha}-1}^{n_{\alpha}}\left(i_{n_{\alpha}}-1, \cdots, i_{t+1}-1, i_{t}, \cdots, i_{1}\right) & \text { if } \quad i_{t+1} \geq s>i_{t}+1, \\
1 & \text { if } \quad i_{t+1} \geq s=i_{t}+1 .\end{cases}
\end{aligned}
$$

Proof. We prove the above result by induction. First, since

$\xi_{n-n_{\alpha}}^{0}\left(i_{n_{\alpha}}, \cdots, i_{1}\right)=s_{n-1} \cdots s_{i_{n_{\alpha}+1}} s_{i_{n_{\alpha}}-1} \cdots s_{i_{t+1}+1} s_{i_{t+1}-1} \cdots s_{i_{t}+1} s_{i_{t}-1} \cdots s_{0} \sigma_{n_{\alpha}}$

by the simplicial identities, we have

$$
\begin{aligned}
& d_{s} \xi_{n-n_{\alpha}}^{0}\left(i_{n_{\alpha}}, \cdots, i_{t+1}-1, i_{t}, \cdots, i_{1}\right) \\
& = \begin{cases}\xi_{n-n_{\alpha}-1}^{0}\left(i_{n_{\alpha}}-1, \cdots, i_{t+1}-1, i_{t}, \cdots, i_{1}\right) & \text { if } \quad i_{t+1} \geq s>i_{t}+1 \\
1 & \text { or } \quad i_{t+1}>s \geq i_{t}+1, \\
1 & \text { if } \quad i_{t+1}=s=i_{t}+1 .\end{cases}
\end{aligned}
$$

Inductively, for any $0 \leq j<n_{\alpha}$, we suppose that $\xi_{n-n_{\alpha}}^{j}\left(i_{n_{\alpha}}, \cdots, i_{1}\right)$ satisfies

(A) If $j<t$, then

$$
\begin{aligned}
& d_{s} \xi_{n-n_{\alpha}}^{j}\left(i_{n_{\alpha}}, \cdots, i_{t+1}-1, i_{t}, \cdots, i_{1}\right) \\
& = \begin{cases}\xi_{n-n_{\alpha}-1}^{j}\left(i_{n_{\alpha}}-1, \cdots, i_{t+1}-1, i_{t}, \cdots, i_{1}\right) & \text { if } i_{t+1} \geq s>i_{t}+1 \\
1 & \text { or } i_{t+1}>s \geq i_{t}+1, \\
1 & \text { if } i_{t+1}=s=i_{t}+1 .\end{cases}
\end{aligned}
$$

(B) If $j \geq t$, then

$$
\begin{aligned}
& d_{s} \xi_{n-n_{\alpha}}^{j}\left(i_{n_{\alpha}}, \cdots, i_{t+1}-1, i_{t}, \cdots, i_{1}\right) \\
& = \begin{cases}\xi_{n-n_{\alpha}-1}^{j}\left(i_{n_{\alpha}}-1, \cdots, i_{t+1}-1, i_{t}, \cdots, i_{1}\right) & \text { if } \quad i_{t+1} \geq s>i_{t}+1, \\
1 & \text { if } \quad i_{t+1} \geq s=i_{t}+1 .\end{cases}
\end{aligned}
$$

We can see that for $j=0$, if $j<t$, then (A) is satisfied. If $j \geq t$, which shows that $t=j=0$, only one case appears, that is, $i_{1} \geq s>i_{0}+1=0$, while the other case $i \geq s=i_{0}+1=0$ does not appear due to $s>0$. This shows that (B) is satisfied. Hence the first step of our induction works.

For $\left\{\xi_{n-n_{\alpha}}^{j+1}\left(i_{n_{\alpha}}, \cdots, i_{1}\right) \mid n=i_{n_{\alpha}+1}>n-1 \geq i_{n_{\alpha}}>\cdots>i_{1}>i_{0}=-1\right\}$, recall that

$$
\begin{aligned}
& \xi_{n-n_{\alpha}}^{j+1}\left(i_{n_{\alpha}}, \cdots, i_{1}\right)= \\
& \left\{\begin{array}{lll}
\xi_{n-n_{\alpha}}^{j}\left(i_{n_{\alpha}}, \cdots, i_{j}, \cdots, i_{1}\right) & \text { if } i_{j+2}=i_{j+1}+1, \\
\xi_{n-n_{\alpha}}^{j}\left(i_{n_{\alpha}}, \cdots, i_{j+1}, \cdots, i_{1}\right) \xi_{n-n_{\alpha}}^{j}\left(i_{n_{\alpha}}, \cdots, i_{j+1}+1, \cdots, i_{1}\right)^{-1} & \text { if } & i_{j+2}>i_{j}+1 .
\end{array}\right.
\end{aligned}
$$

Stated as above, for any $1 \leq s \leq n$, there is some $0 \leq t \leq n_{\alpha}$ such that $i_{t+1} \geq s \geq i_{t}+1$. If $t=j+1$, we argue in the following three cases.

Case 1. $i_{j+2} \geq s \geq i_{j+1}+1$.

If $i_{j+2}-1>i_{j+1}+1$, then we have

$$
\begin{aligned}
& d_{s} \xi_{n-n_{\alpha}}^{j+1}\left(i_{n_{\alpha}}, \cdots, i_{j+2}, i_{j+1}, \cdots, i_{1}\right) \\
& =d_{s} \xi_{n-n_{\alpha}}^{j}\left(i_{n_{\alpha}}, \cdots, i_{j+2}, i_{j+1}, \cdots, i_{1}\right) d_{s} \xi_{n-n_{\alpha}}^{j}\left(i_{n_{\alpha}}, \cdots, i_{j+2}, i_{j+1}+1, \cdots, i_{1}\right)^{-1} \\
& =\xi_{n-n_{\alpha}-1}^{j}\left(i_{n_{\alpha}}-1, \cdots, i_{j+2}-1, i_{j+1}, \cdots, i_{1}\right) \xi_{n-n_{\alpha}-1}^{j}\left(i_{n_{\alpha}}-1, \cdots, i_{j+2}-1, i_{j+1}\right. \\
& \left.\quad \quad+1, \cdots, i_{1}\right)^{-1} \\
& =\xi_{n-n_{\alpha}-1}^{j+1}\left(i_{n_{\alpha}}-1, \cdots, i_{j+2}-1, i_{j+1}, \cdots, i_{1}\right) .
\end{aligned}
$$


If $i_{j+2}-1=i_{j+1}+1$, then we have

$d_{s} \xi_{n-n_{\alpha}}^{j+1}\left(i_{n_{\alpha}}, \cdots, i_{j+2}, i_{j+1}, \cdots, i_{1}\right)$

$=d_{s} \xi_{n-n_{\alpha}}^{j}\left(i_{n_{\alpha}}, \cdots, i_{j+2}, i_{j+1}, \cdots, i_{1}\right) d_{s} \xi_{n-n_{\alpha}}^{j}\left(i_{n_{\alpha}}, \cdots, i_{j+2}, i_{j+1}+1, \cdots, i_{1}\right)^{-1}$

$=\xi_{n-n_{\alpha}-1}^{j}\left(i_{n_{\alpha}}-1, \cdots, i_{j+2}-1, i_{j+1}, \cdots, i_{1}\right)$

$=\xi_{n-n_{\alpha}-1}^{j+1}\left(i_{n_{\alpha}}-1, \cdots, i_{j+2}-1, i_{j+1}, \cdots, i_{1}\right)$.

Case 2. $i_{j+2}=s=i_{j+1}+1$. We have

$$
d_{s} \xi_{n-n_{\alpha}}^{j+1}\left(i_{n_{\alpha}}, \cdots, i_{j+2}, i_{j+1}, \cdots, i_{1}\right)=d_{s} \xi_{n-n_{\alpha}}^{j}\left(i_{n_{\alpha}}, \cdots, i_{j+2}, i_{j+1}, \cdots, i_{1}\right)=1 .
$$

Case 3. $i_{j+2}>s=i_{j+1}+1$.

Since $s=i_{j+1}+1>i_{j}+1$, we have

$$
\begin{aligned}
& d_{s} \xi_{n-n_{\alpha}}^{j+1}\left(i_{n_{\alpha}}, \cdots, i_{j+2}, i_{j+1}, \cdots, i_{1}\right) \\
& =d_{s} \xi_{n-n_{\alpha}}^{j+1}\left(i_{n_{\alpha}}, \cdots, i_{j+2}, i_{j+1}, \cdots, i_{1}\right) d_{s} \xi_{n-n_{\alpha}}^{j}\left(i_{n_{\alpha}}, \cdots, i_{j+2}, i_{j+1}+1, \cdots, i_{1}\right)^{-1} \\
& =\xi_{n-n_{\alpha}-1}^{j}\left(i_{n_{\alpha}}-1, \cdots, i_{j+2}-1, i_{j+1}, \cdots, i_{1}\right) \xi_{n-n_{\alpha}-1}^{j}\left(i_{n_{\alpha}}-1, \cdots, i_{j+2}-1, i_{j+1},\right. \\
& \left.\quad \quad \cdots, i_{1}\right)^{-1} \\
& =1 .
\end{aligned}
$$

Similarly, we can show that for $t>j+1$, (A) is satisfied; for $t<j+1$, (B) is satisfied. So after finite steps, the desired result follows. This finishes our proof.

Corollary 3.3. The face operator $d_{s}$ in Proposition 3.2 is a projection homomorphism up to an isomorphism.

Proof. Let

$$
\begin{aligned}
S= & \left\{\xi_{n-n_{\alpha}}^{n_{\alpha}}\left(i_{n_{\alpha}}, \cdots, i_{1}\right) \mid n-1 \geq i_{n_{\alpha}}>\cdots>i_{1} \geq 0\right\}, \\
T_{s}= & \left\{\xi_{n-n_{\alpha}}^{n_{\alpha}}\left(i_{n_{\alpha}}, \cdots, i_{1}\right) \mid n-1 \geq i_{n_{\alpha}}>\cdots>i_{1} \geq 0, s \neq i_{t} \text { for any } 1 \leq t \leq n_{\alpha}\right\}, \\
T_{s}^{\prime}= & \left\{\xi_{n-n_{\alpha}}^{n_{\alpha}}\left(i_{n_{\alpha}}-1, \cdots, i_{t+1}-1, i_{t}, \cdots, i_{1}\right) \mid n-1 \geq i_{n_{\alpha}}>\cdots>i_{1} \geq 0, i_{t+1}-1,\right. \\
& \left.s>i_{t}\right\} .
\end{aligned}
$$

Then we have the following commutative diagram:

where $\bar{d}_{s}$ is defined by

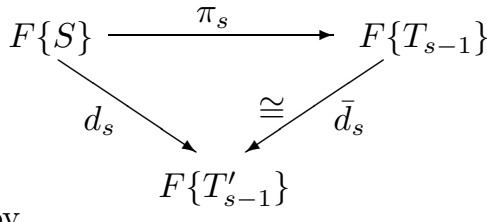

$$
\bar{d}_{s} \xi_{n-n_{\alpha}}^{n_{\alpha}}\left(i_{n_{\alpha}}, \cdots, i_{1}\right)=\xi_{n-n_{\alpha}}^{n_{\alpha}}\left(i_{n_{\alpha}}-1, \cdots, i_{t+1}-1, i_{t}, \cdots, i_{1}\right)
$$

for $i_{t+1} \geq s>i_{t}+1$. Then the assertion follows from the commutative diagram.

Proposition 3.4. For any $0 \leq j \leq n$, there must be $0 \leq t \leq n_{\alpha}$ such that $i_{t+1} \geq j \geq i_{t}+1$. Then we have

$$
\begin{aligned}
& s_{j} \xi_{n-n_{\alpha}}^{n_{\alpha}}\left(i_{n_{\alpha}}, \cdots, i_{t+1}, i_{t}, \cdots, i_{1}\right) \\
& =\left\{\begin{array}{c}
\xi_{n \alpha-n_{\alpha}+1}^{n_{\alpha}}\left(i_{n_{\alpha}}+1, \cdots, i_{t+1}+1, i_{t}, \cdots, i_{1}\right) \quad \text { if } i_{t+1} \geq s>i_{t}+1, \\
\xi_{n-n_{\alpha}+1}^{n_{\alpha}}\left(i_{n_{\alpha}}, \cdots, i_{1}\right) \xi_{n-n_{\alpha}+1}^{n_{\alpha}}\left(i_{n_{\alpha}}+1, \cdots, i_{t}+1, i_{t-1}, \cdots, i_{1}\right)
\end{array}\right. \\
& \text { if } i_{t+1} \geq s=i_{t}+1 .
\end{aligned}
$$


Proof. The proof is similar to that of Proposition 3.2 by a slight modification.

\section{Proof of Theorem 1.2}

Given the wedge of spheres $\bigvee_{\alpha \in J} S^{n_{\alpha}}$ with index set $J$ and $n-1>\max \left\{n_{\alpha} \mid \alpha \in\right.$ $J\} \geq 1$, the Milnor construction of $\bigvee_{\alpha \in J} S^{n_{\alpha}}$ is $F\left(\bigvee_{\alpha \in J} S^{n_{\alpha}}\right)$ whose geometric realization is homotopic equivalent to $\Omega \Sigma \bigvee_{\alpha \in J} S^{n_{\alpha}}$. Our combinatorial description of $\pi_{n+1}\left(\bigvee_{\alpha \in J} S^{n_{\alpha}+1}\right)$ will follow from our general results on simplicial groups and free groups in Section 2 together with determination of the Moore chain complex $\left(\mathscr{N} F\left(\bigvee_{\alpha \in J} S^{n_{\alpha}}\right), d_{0}\right)$.

By Proposition 3.1, we can see that

$$
F\left(\bigvee_{\alpha \in J} S^{n_{\alpha}}\right)_{n}=F\left\{\xi_{n-n_{\alpha}}^{n_{\alpha}}\left(i_{n_{\alpha}}, \cdots, i_{1}\right)^{(\alpha)} \mid n-1 \geq i_{n_{\alpha}}>\cdots>i_{1} \geq 0, \alpha \in J\right\}
$$

Let

$$
\begin{aligned}
S & =\left\{\xi_{n-n_{\alpha}}^{n_{\alpha}}\left(i_{n_{\alpha}}, \cdots, i_{1}\right)^{(\alpha)} \mid n-1 \geq i_{n_{\alpha}}>\cdots>i_{1} \geq 0, \alpha \in J\right\}, \\
T_{j} & =\left\{\xi_{n-n_{\alpha}}^{n_{\alpha}}\left(i_{n_{\alpha}}, \cdots, i_{1}\right)^{(\alpha)} \mid n-1 \geq i_{n_{\alpha}}>\cdots>i_{1} \geq 0, s \neq i_{t} \text { for any } 1 \leq t\right. \\
& \left.\leq n_{\alpha}, \alpha \in J\right\},
\end{aligned}
$$

where $0 \leq j \leq n-1$. According to Corollary 3.3, for $1 \leq s \leq n$, the face operator $d_{s}$ on $F\left(\bigvee_{\alpha \in J} S^{n_{\alpha}}\right)_{n}$ is a projection homomorphism

$$
\pi_{s}: F\{S\} \longrightarrow F\left\{T_{s-1}\right\} .
$$

Since $S=\bigcup_{j=0}^{n-1} T_{j}$, by Theorem 2.3 , we get

Lemma 4.1. $\mathscr{N} F\left(\bigvee_{\alpha \in J} S^{n_{\alpha}}\right)_{n}=\bigcap_{j=1}^{n} \operatorname{Ker}\left(\pi_{j}\right)=F\left\{A\left(T_{0}, \cdots, T_{n-1}\right)\right\}$.

Let $\mathscr{C}_{n}$ be the subgroup of $F\left(\bigvee_{\alpha \in J} S^{n_{\alpha}}\right)_{n}$ generated by all of the commutators of the form

$$
\beta^{t}\left(\xi_{n-n_{\alpha_{1}}}^{n_{\alpha_{1}}}\left(i_{n_{\alpha_{1}}}^{(1)}, \cdots, i_{1}^{(1)}\right)^{\left(\alpha_{1}\right) \varepsilon_{1}}, \cdots, \xi_{n-n_{\alpha_{t}}}^{n_{\alpha_{t}}}\left(i_{n_{\alpha_{t}}}^{(t)}, \cdots, i_{1}^{(t)}\right)^{\left(\alpha_{t}\right) \varepsilon_{t}}\right),
$$

where

(1) $\varepsilon_{j}= \pm 1$ for $1 \leq j \leq t$;

(2) $n-1 \geq i_{n_{\alpha_{s}}}^{(s)}>\cdots>i_{1}^{(s)} \geq 0$ for $1 \leq s \leq t$;

(3) any of $\{0,1, \cdots, n-1\}$ appears at least once in $\left\{i_{n_{\alpha_{1}}}^{(1)}, \cdots, i_{1}^{(1)}, \cdots, i_{n_{\alpha_{t}}}^{(t)}\right.$, $\left.\cdots, i_{t}^{(1)}\right\}$;

(4) for $t \geq\left[n / \max \left\{n_{\alpha} \mid \alpha \in J\right\}\right]$, $\beta^{t}$ runs over all of the commutator bracket arrangements of weight $t$.

Lemma 4.2. $\mathscr{C}_{n}=\mathscr{N} F\left(\bigvee_{\alpha \in J} S^{n_{\alpha}}\right)_{n}=F\left\{A\left(T_{0}, \cdots, T_{n-1}\right)\right\}$.

Proof. The proof of this lemma is similar to that of Theorem 4.5 in [4].

Proof of Theorem 1.2. Note that $F\left(\bigvee_{\alpha \in J} S^{n_{\alpha}}\right)$ is a free simplicial group. We can easily see that $F\left(\bigvee_{\alpha \in J} S^{n_{\alpha}}\right)$ is $\left(\max \left\{n_{\alpha} \mid \alpha \in J\right\}+1\right)$-centerless. Then by Theorem 2.2 and Lemma 4.2, we get

$$
\begin{aligned}
\pi_{n+1}\left(\bigvee_{\alpha \in J} S^{n_{\alpha}+1}\right) & =\pi_{n}\left(F\left(\bigvee_{\alpha \in J} S^{n_{\alpha}}\right)\right) \\
& =C\left(F\{S\} / \mathscr{B} F\left(\bigvee_{\alpha \in J} S^{n_{\alpha}}\right)_{n}\right)=C\left(F\{S\} / d_{0} \mathscr{C}_{n+1}\right) .
\end{aligned}
$$


In order to compute $d_{0} \mathscr{C}_{n+1}$, we first inductively compute $d_{0} \xi_{n-n_{\alpha}+1}^{n_{\alpha}}\left(i_{n_{\alpha}}, \cdots, i_{1}\right)$ for any $\xi_{n-n_{\alpha}+1}^{n_{\alpha}}\left(i_{n_{\alpha}}, \cdots, i_{1}\right) \in\left(F S^{n_{\alpha}}\right)_{n+1}$ with $n \geq i_{n_{\alpha}}>\cdots>i_{1} \geq 0$.

Since

$$
\left.\xi_{n-n_{\alpha}}^{0}\left(i_{n_{\alpha}}, \cdots, i_{1}\right)\right)=s_{n} \cdots s_{i_{n_{\alpha}}+1} s_{i_{n_{\alpha}}-1} \cdots s_{i_{t}+1} s_{i_{t}-1} \cdots s_{i_{1}+1} s_{i_{1}-1} \cdots s_{0} \sigma_{n_{\alpha}},
$$

we have

$$
d_{0} \xi_{n-n_{\alpha}+1}^{0}\left(i_{n_{\alpha}}, \cdots, i_{1}\right)=\xi_{n-n_{\alpha}}^{0}\left(i_{n_{\alpha}}-1, \cdots, i_{1}-1\right) .
$$

Inductively for $0 \leq j<n_{\alpha}-1$, suppose that we have

$$
d_{0} \xi_{n-n_{\alpha}+1}^{j}\left(i_{n_{\alpha}}, \cdots, i_{1}\right)=\xi_{n-n_{\alpha}}^{j}\left(i_{n_{\alpha}}-1, \cdots, i_{1}-1\right) .
$$

Recall that

$$
\begin{aligned}
\xi_{n-n_{\alpha}+1}^{j+1}\left(i_{n_{\alpha}}, \cdots, i_{j+1}, \cdots, i_{1}\right) & \\
= & \left\{\begin{array}{cc}
\xi_{n-n_{\alpha}+1}^{j}\left(i_{n_{\alpha}}, \cdots, i_{j+1}, \cdots, i_{1}\right) & \text { if } i_{j+2}=i_{j+1}+1, \\
\xi_{n-n_{\alpha}+1}^{j}\left(i_{n_{\alpha}}, \cdots, i_{j+1}, \cdots, i_{1}\right) \xi_{n-n_{\alpha}+1}^{j}\left(i_{n_{\alpha}}, \cdots,\right. & \left.i_{j+1}+1, \cdots, i_{1}\right)^{-1} \\
\text { if } i_{j+2}>i_{j+1}+1 .
\end{array}\right.
\end{aligned}
$$

We can also easily verify that

$$
d_{0} \xi_{n-n_{\alpha}+1}^{j+1}\left(i_{n_{\alpha}}, \cdots, i_{j+1}, \cdots, i_{1}\right)=\xi_{n-n_{\alpha}}^{j+1}\left(i_{n_{\alpha}}-1, \cdots, i_{j+1}-1, \cdots, i_{1}-1\right) .
$$

So after finite steps, we have

$$
d_{0} \xi_{n-n_{\alpha}+1}^{n_{\alpha}}\left(i_{n_{\alpha}}, \cdots, i_{j+1}, \cdots, i_{1}\right)=\xi_{n-n_{\alpha}}^{n_{\alpha}}\left(i_{n_{\alpha}}-1, \cdots, i_{j+1}-1, \cdots, i_{1}-1\right) .
$$

When $i_{1}=0$, we denote $d_{0} \xi_{n-n_{\alpha}+1}^{n_{\alpha}}\left(i_{n_{\alpha}}, \cdots, i_{j+1}, \cdots, 0\right)$ by $\xi_{n-n_{\alpha}}^{n_{\alpha}}\left(i_{n_{\alpha}}-1, \cdots\right.$, $\left.i_{j+1}-1, \cdots,-1\right)$.

According to the above results, given any

$$
\xi=\beta^{t}\left(\xi_{n-n_{\alpha_{1}}}^{n_{\alpha_{1}}}\left(i_{n_{\alpha_{1}}}^{(1)}, \cdots, i_{1}^{(1)}\right)^{\left(\alpha_{1}\right) \varepsilon_{1}}, \cdots, \xi_{n-n_{\alpha_{t}}}^{n_{\alpha_{t}}}\left(i_{n_{\alpha_{t}}}^{(t)}, \cdots, i_{1}^{(t)}\right)^{\left(\alpha_{t}\right) \varepsilon_{t}}\right) \in \mathscr{C}_{n+1},
$$

we have

$$
\begin{aligned}
d_{0} \xi=\beta^{t}\left(\xi_{n-n_{\alpha_{1}}}^{n_{\alpha_{1}}}\left(i_{n_{\alpha_{1}}}^{(1)}-1, \cdots, i_{1}^{(1)}-1\right)^{\left(\alpha_{1}\right) \varepsilon_{1}},\right. \\
\left.\cdots, \xi_{n-n_{\alpha_{t}}}^{n_{\alpha_{t}}}\left(i_{n_{\alpha_{t}}}^{(t)}-1, \cdots, i_{1}^{(t)}-1\right)^{\left(\alpha_{t}\right) \varepsilon_{t}}\right) \in \mathscr{B} F\left(\vee_{\alpha \in J} S^{n_{\alpha}}\right)_{n} .
\end{aligned}
$$

By the definition of $\mathscr{C}_{n+1}$, our main theorem follows.

When the index set $J$ contains only one element $\alpha$, we have the following corollary.

Corollary 4.3. For $n-1>n_{\alpha} \geq 1$, the homotopy group $\pi_{n+1}\left(S^{n_{\alpha}+1}\right)$ is isomorphic to the center of the group

$$
F\left\{\xi_{n-n_{\alpha}}^{n_{\alpha}}\left(i_{n_{\alpha}}, \cdots, i_{1}\right) \mid n-1 \geq i_{n_{\alpha}}>\cdots>i_{1} \geq 0\right\}
$$

modulo the normal subgroup generated by

$$
\beta^{t}\left(\xi_{n-n_{\alpha}}^{n_{\alpha}}\left(i_{n_{\alpha}}^{(1)}, \cdots, i_{1}^{(1)}\right)^{\varepsilon_{1}}, \cdots, \xi_{n-n_{\alpha}}^{n_{\alpha}}\left(i_{n_{\alpha}}^{(t)}, \cdots, i_{1}^{(t)}\right)^{\varepsilon_{t}}\right),
$$

where

(1) $\varepsilon_{j}= \pm 1$ for $1 \leq j \leq t$

(2) $n-1 \geq i_{n_{\alpha}}^{(s)}>\cdots>i_{1}^{(s)} \geq-1$ for $1 \leq s \leq t$;

(3) any of $\{-1,0, \cdots, n-1\}$ appears at least once in $\left\{i_{n_{\alpha}}^{(1)}, \cdots, i_{1}^{(1)}, \cdots, i_{n_{\alpha}}^{(t)}\right.$, $\left.\cdots, i_{t}^{(t)}\right\}$;

(4) for each $t \geq\left[n / n_{\alpha}\right], \beta^{t}$ runs over all of the commutator bracket arrangements of weight $t$. 
In Theorem 1.2, if $n_{\alpha}=1$ for all $\alpha \in J$, then $F\left(\bigvee_{\alpha \in J} S^{1}\right)_{n}$ is freely generated by

$$
\left\{y_{i}^{\alpha}=\xi_{n-1}^{1}(i)^{(\alpha)} \mid n-1 \geq i \geq 0, \alpha \in J\right\} .
$$

Since

$$
\begin{aligned}
y_{-1}^{(\alpha)} & =\xi_{n-1}^{1}(-1)^{(\alpha)}=d_{0} \xi_{n}^{1}(0)^{(\alpha)}=d_{0} \xi_{n}^{0}(0)^{(\alpha)}\left(d_{0} \xi_{n}^{0}(1)^{(\alpha)}\right)^{-1} \\
& =\left(\xi_{n-1}^{0}(0)^{(\alpha)}\right)^{-1}=\left(\xi_{n-1}^{1}(0)^{(\alpha)} \xi_{n-1}^{1}(1)^{(\alpha)} \cdots \xi_{n-1}^{1}(n-1)^{(\alpha)}\right)^{-1} \\
& =\left(y_{0}^{(\alpha)} y_{1}^{(\alpha)} \cdots y_{n-1}^{(\alpha)}\right)^{-1},
\end{aligned}
$$

we can deduces J. Wu's result as follows.

Corollary 4.4 (4]). For $n>2, \pi_{n+1}\left(\bigvee_{\alpha \in J} S^{2}\right)$ is the center of the group $G(n+1)$, where $G(n+1)$ is the free group

$$
F\left\{y_{i}^{(\alpha)} \mid n-1 \geq i \geq 0, \alpha \in J\right\}
$$

modulo the normal subgroup generated by $\beta^{t}\left(y_{i_{1}}^{\left(\alpha_{1}\right) \varepsilon_{1}}, \cdots, y_{i_{t}}^{\left(\alpha_{t}\right) \varepsilon_{t}}\right)$, where

(1) $\varepsilon_{j}= \pm 1$ for $1 \leq j \leq t$;

(2) $n-1 \geq i_{s} \geq-1$ for $1 \leq s \leq t$;

(3) any of $\{-1,0, \cdots, n-1\}$ appears at least once in $\left\{i_{1}, \cdots, i_{t}\right\}$;

(4) for $t \geq n+1, \beta^{t}$ runs over all commutator bracket arrangements of weight $t$.

Remark. According to Corollary 4.4, J. Wu defined an action of Artin braid groups $B_{n}$ on the group $G(n)$ (see Corollary 4.4) and proved that the homotopy group $\pi_{n} S^{2}$ is actually given by the fixed set of the pure braid group $P_{n}$ on $G(n)$ [ 8 , Theorem 1.2] (refer to [9] on braid groups). More recently, A. J. Berrick, F. R. Cohen, Y. L. Wong and $\mathrm{J} . \mathrm{Wu}\left[10\right.$ ] established a deeper connection of $\pi_{*} S^{2}$ with configuration spaces and braid groups. These results are all based on J. Wu's original work, that is, Corollary 4.4. However it remains to be seen whether new knowledge about the unidentified higher homotopy groups of spheres can be obtained through the methods of [10]. We hope that our work gives a good start.

\section{REFERENCES}

1. D. M. Kan, On c.s.s. complexes, Amer. J. Math., 79(1957), 449-476. MR0090047 (19:759e)

2. E. B. Curtis, Simplicial homotopy theory, Advances in Math. 6, 1971, 107-209. MR0279808 (43:5529)

3. D. M. Kan, A combinatorial definition of homotopy groups, Ann. of Math. (2), 67(1958), 282-312. MR0111032 (22:1897)

4. J. Wu, Combinatorial descriptions of homotopy groups of certain spaces, Math. Proc. Camb. Philos. Soc., 130(2001), 489-513. MR.1816806 (2003e:55014)

5. J. P. May, Simplicial objects in algebraic topology, Math. Studies 11, van Nostrand, Princeton, NJ, Toronto, London, 1967. MR0222892 (36:5942)

6. J. Milnor, On the construction FK, Algebraic Topology-A Student Guide, by J. F. Adams, LMS Lec. Note. Ser. 4, Cambridge Univ. Press, 1972, 119-136.

7. W. Magnus, A. Karrass and D. Solitar, Combinatorial group theory, Interscience Publishers, New York, London, Sydney, 1966. MR 0207802 (34:7617)

8. J. Wu, A braided simplicial group, Proc. London Math. Soc. (3), 84(2002), 645-662. MR.1888426 (2003e:20041) 
9. J. S. Birman, Braids, links and mapping class groups, Annals of Math. Studies 82, Princeton University Press; University of Tokyo Press, 1974. MR 0375281 (51:11477)

10. A. J. Berrick, F. R. Cohen, Y. L. Wong and J. Wu, Configurations, braids and homotopy groups, J. Amer. Math. Soc., 19(2006), 265-326. MR2188127 (2007e:20073)

School of Mathematical Sciences, Nankai University, Tianjin 300071, People's Republic of China

Current address: School of Mathematics, The University of Manchester, Oxford Road, Manchester, M13 9PL, United Kingdom

E-mail address: Hao.Zhao@manchester.ac.uk

School of Mathematical Sciences, Nankai University, Tianjin 300071, People's Republic of China

E-mail address: xjwang@nankai.edu.cn 